\title{
Maternal and neonatal outcomes following in vitro fertilization: A cohort study in Romania
}

\author{
ANDREEA MĂDĂLINA BĂNICĂ $\breve{~}^{1,2}$, SIMONA DANIELA POPESCU ${ }^{1,2}$ and SIMONA VLĂDĂREANU ${ }^{1,2}$ \\ ${ }^{1}$ Department 13 - Obstetrics-Gynecology, Faculty of Medicine, 'Carol Davila' University of Medicine and Pharmacy, \\ 020021 Bucharest; ${ }^{2}$ Department of Neonatology, 'Elias’ University Emergency Hospital, 011461 Bucharest, Romania
}

Received September 3, 2021; Accepted October 5, 2021

DOI: $10.3892 /$ etm.2021.10956

\begin{abstract}
Although in recent years the number of pregnancies obtained through in vitro fertilization (IVF) has increased significantly, a higher incidence of complications has been identified in this group. The widespread development and use of IVF has led to an increasing rate of multiple pregnancies and thus their associated complications. However, whether these complications occur due to assisted reproduction or infertility problems remains to be elucidated. In the present study, a comparison was made of IVF pregnancies with spontaneous conception, and the risk of obstetric and neonatal complications was assessed. An ambispective observational cohort study was carried out between January 2017 and October 2021 at Elias University Emergency Hospital, Romania. The exposed cohort included 132 IVF pregnancies and the control cohort included 157 spontaneous pregnancies. The IVF group included 110 pregnancies with fresh embryo-transfer and 22 pregnancies with cryopreserved embryo-transfer. Obstetric, perinatal and neonatal complications were analysed by multivariable logistic analysis. The results showed that, IVF pregnancies had a higher risk of pregnancy-induced hypertension $(\mathrm{OR}=6.42$, 95\% CI=1.72-23.92) and placental abnormalities (OR=5.49, 95\% CI=1.07-28.17). Neonates obtained through IVF had a higher risk of prematurity $(\mathrm{OR}=6.52,95 \% \mathrm{CI}=2.99-14.20)$, low birth weight ( $\mathrm{LBW})(\mathrm{OR}=17.18,95 \% \mathrm{CI}=7.06-41.87)$, small for gestational age $(\mathrm{OR}=4.10,95 \% \mathrm{CI}=1.95-8.59)$ and were more
\end{abstract}

Correspondence to: Dr Andreea Mădălina Bănică, Department of Neonatology, 'Elias' University Emergency Hospital, 17 Mărăști Boulevard, 011461 Bucharest, Romania

E-mail: aandreeabanica@gmail.com

Abbreviations: ART, assisted reproductive techniques; IVF, in vitro fertilization; ICSI, intracytoplasmic sperm injection; LBW, low birth weight; VLBW, very low birth weight; SGA, small for gestational age; IUGR, intrauterine growth restriction; LGA, large for gestational age; NICU, Neonatal Intensive Care Unit; RDS, respiratory distress syndrome; Nasal CPAP, nasal continuous positive airway pressure; e-SET, elective single embryo-transfer

Key words: in vitro fertilization, obstetric complications, neonatal complications, elective single embryo-transfer, multiple pregnancies frequently hospitalized in the Neonatal Intensive Care Unit (NICU) $(\mathrm{OR}=11.91,95 \% \mathrm{CI}=5.72-24.81)$. Nulliparous women were associated with an increased risk of NICU admission $(\mathrm{OR}=0.46,95 \% \mathrm{CI}=0.25-0.88)$ and risk of $\mathrm{LBW}(\mathrm{OR}=0.37$, $95 \% \mathrm{CI}=0.19-0.75)$. Maternal age $\geq 35$ years had no influence as a confounding variable. In conclusion, this cohort study showed that IVF pregnancies have a higher risk of obstetric and neonatal complications than pregnancies obtained by spontaneous conception.

\section{Introduction}

Recently, the use of assisted reproduction techniques (ARTs) has increased worldwide. In Romania, in 2016, according to ESHRE records, $0.7 \%$ of births were obtained through assisted reproduction (1).

The benefits of treating infertility are known. Nevertheless, previous findings have shown that ARTs increase the risk of obstetric and perinatal complications, even after limiting the analysis to singleton pregnancies (2-4).

It is not possible to establish with certainty whether the complications are determined by the techniques used [e.g., in vitro fertilization or intracytoplasmic sperm insemination (ICSI)], the culture medium used, cryopreservation techniques, or embryo selection methods. Individual factors such as the etiology of infertility or maternal characteristics (age, parity) should also be taken into consideration (5). Other studies have shown the opposite result, namely, that pregnancies obtained through in vitro fertilization (IVF)/intracytoplasmic sperm injection (ICSI) are not correlated with the occurrence of these complications in pregnancy. This hypothesis has been supported by studies conducted on populations of subfertile women who obtained a pregnancy without the use of ART, but who nevertheless presented complications during pregnancy. Marino et al demonstrated in a cohort study conducted between 1986 and 2002 that, subfertile women, diagnosed with infertility but untreated, are at risk of perinatal complications such as prematurity, low birth weight (LBW) and very low birth weight (VLBW) (6). After adjusting for confounding factors, Jaques et al demonstrated in a cohort study that subfertile women who become pregnant spontaneously are at risk for obstetric and perinatal complications compared to the general population. These complications included 
pregnancy-induced hypertension, preeclampsia, perinatal death, but also an increased risk of prematurity and LBW (7).

Researchers believe that the increased number of multiple pregnancies in the ART group may be another cause for the increased incidence of complications in this population. Simultaneously, it has been shown that complications also occur in singleton pregnancies obtained through IVF (8).

In the present study, a comparison of pregnancies obtained through IVF was made with those obtained spontaneously to identify the risk of obstetric and neonatal complications between the two groups in Romania.

\section{Patients and methods}

Data collection. This is an ambispective study (retrospective, between January 2017 and September 2018, and prospective, between October 2018 and March 2021) conducted at Elias University Emergency Hospital, Bucharest (Romania). The pregnancies obtained by IVF were grouped 1:1 with the pregnancies obtained by spontaneous conception. The study included 157 pregnancies obtained through IVF, from which were excluded pregnancies obtained from oocyte donation $(n=19)$, sperm donation $(n=1)$ and pregnancies with incomplete retrospective data $(n=5)$. Thus, the final cohort was composed of 132 pregnancies obtained through IVF and 157 pregnancies obtained spontaneously. The two groups were compared for the risk of obstetric and neonatal complications. Depending on the type of embryo transferred, the IVF group was divided into two subgroups: fresh and frozen embryo.

The present study was approved by the Ethics Committee of Elias University Emergency Hospital (approval no.: 7017). Informed consent was obtained from the pregnant women included in the study. Data were retrospectively obtained from observation sheets and prospectively, anamnestic and from observation sheets during hospitalization. Patients with chronic conditions (chronic arterial hypertension, diabetes mellitus, cardiovascular disease or fetal abnormalities) were excluded because they could have been considered important confounders.

Variables. The maternal variables studied included complications in pregnancy (pregnancy induced-hypertension, gestational diabetes and placental abnormalities such as placenta praevia and placental abruption) and type of pregnancy (singleton/multiple).

Neonatal variables included gestational age (weeks), changes in birth weight (grams) [LBW, small for gestational age (SGA), large for gestational age (LGA), high birth weight, intrauterine growth restriction (IUGR)]. Using their birth weight, newborns can be classified as having a low birth weight which is $<2,500 \mathrm{~g}$ or as having a high birth weight which is between 4,000 and 4,500 g. Newborns can be also classified by intrauterine growth and gestational age. In this regard, we defined small for gestational age as a birth weight below the 10th percentile for gestational age. Large for gestational age was defined as a birth weight above the 90th percentile for gestational age. Intrauterine growth restriction was defined as a reduction in the expected fetal growth and was usually an ultrasound finding. The incidence of neonatal complications [respiratory distress syndrome (RDS), apnea of prematurity, neonatal arterial hypotension and congenital anomalies], Apgar score at $1 \mathrm{~min}$, admission in NICU, the need for respiratory support by oxygen therapy, non-invasive ventilation with a T-piece system and nasal CPAP or mechanical ventilation were analyzed. The need for parenteral nutrition, antibiotic therapy, vasoactive medication and blood transfusions was also analyzed.

Statistical analysis. The analysis of qualitative variables was performed using the Chi-square independence test (Pearson). Maternal age as a numerical variable was analyzed using ANOVA parametric test as the values had a Gaussian distribution for each of the three subgroups compared. A significant difference was detected globally thus, the analysis was continued using the Fisher LSD post hoc test to make comparisons between all pairs of groups.

For gestational age and birth weight analysis, the non-parametric Kruskal-Wallis comparison test was used as the distribution of data from the three groups was not Gaussian. Subsequently, a post hoc analysis was performed by the Dunn procedure for multiple pair comparisons.

In addition, a multivariable logistic analysis was performed using maternal age and parity as independent variables to identify the predictive factors for the complications sought and for the association between IVF and spontaneous conception. From this analysis, odd ratios (OR) were calculated with a $95 \%$ confidence interval (CI) and probability (P)-value. The limit for statistical significance was $\mathrm{P}<0.05$. Alpha level was 0.05 for all tests. MS Excel 2019 was used for data analysis.

\section{Results}

Maternal and neonatal characteristics for the IVF/spontaneous conception group. This study included 132 pregnancies obtained through IVF and 157 pregnancies obtained through spontaneous conception, from January 2017 to March 2021. The IVF group comprised 77 singleton pregnancies and 55 multiple pregnancies (26 twin pregnancies and one triplet pregnancy), and the control group consisted of 157 single pregnancies and did not include multiple pregnancies. The IVF group was divided into two subgroups depending on the type of embryos used for transfer: fresh embryo $(n=61$ used for singleton pregnancies and $n=49$ used for multiple pregnancies), and cryopreserved embryo ( $n=16$ used for singleton pregnancies and $n=6$ used for multiple pregnancies). Tables I and II summarize maternal and neonatal characteristics in the IVF and in the spontaneous conception (control) groups.

In the post hoc analysis of maternal age, highly significant differences were observed between the control group and IVF singleton pregnancy group, but also between the control group and the IVF multiple pregnancy group. However, no statistically significant differences were demonstrated between the two subgroups of IVF. In the present study, women who opted for IVF were older than those in the control group (Table I).

Analyzing gestational age and birth weight, the differences found among the three groups were highly significant $(\mathrm{P}<0.0001$, both for gestational age and birth weight). The 
Table I. Maternal and neonatal characteristics for the IVF/spontaneous conception group.

\begin{tabular}{|c|c|c|c|c|c|c|c|c|c|c|c|}
\hline Variable & & Group & No. & Mean & SD & Min & Quartile & $\begin{array}{c}\text { 1st } \\
\text { Median }\end{array}$ & Quartile & $\begin{array}{c}\text { 3rd } \\
\text { Max }\end{array}$ & P-value \\
\hline \multirow{3}{*}{$\begin{array}{l}\text { Maternalage } \\
\text { (years) }\end{array}$} & \multicolumn{2}{|c|}{ Control group } & 157 & 30.55 & 5.20 & 19 & 27 & 31 & 34 & 44 & \\
\hline & IVF & Singleton & 77 & 35.90 & 5.69 & 26 & 31 & 36 & 39 & 50 & $<0.0001^{\mathrm{a}}$ \\
\hline & & Multiple & 55 & 34.80 & 6.33 & 25 & 31 & 35 & 39 & 50 & $<0.0001^{\mathrm{a}}$ \\
\hline \multirow[t]{3}{*}{ GA (weeks) } & \multicolumn{2}{|c|}{ Control group } & 157 & 38.88 & 1.24 & 33 & 39 & 39 & 40 & 42 & \\
\hline & IVF & Singleton & 77 & 37.97 & 1.63 & 33 & 38 & 38 & 39 & 40 & $<0.0001^{\mathrm{b}}$ \\
\hline & & Multiple & 55 & 35.87 & 2.29 & 26 & 35 & 36 & 37 & 38 & $<0.0001^{\mathrm{b}}$ \\
\hline \multirow[t]{3}{*}{$\mathrm{BW}(\mathrm{g})$} & \multicolumn{2}{|c|}{ Control group } & 157 & $3,260.45$ & 416.62 & 1,530 & 3,080 & 3,250 & 3,500 & 4,460 & \\
\hline & \multirow[t]{2}{*}{ IVF } & Singleton & 77 & $3,002.60$ & 658.87 & 240 & 2,670 & 3,090 & 3,340 & 4,550 & $<0.0001^{\mathrm{b}}$ \\
\hline & & Multiple & 55 & $2,289.82$ & 446.74 & 800 & 2,095 & 2,340 & 2,570 & 3,080 & $<0.0001^{\mathrm{b}}$ \\
\hline
\end{tabular}

IVF, in vitro fertilization; GA, gestational age; BW, birth weight; $\mathrm{SD}$, standard deviation. $\mathrm{P}<0.0001$, highly significant. $\mathrm{P}$-values according to ${ }^{\mathrm{a} A N O V A}$ and ${ }^{\mathrm{b}}$ Kruskal Wallis.

Table II. Maternal characteristics for the IVF/spontaneous conception group.

\begin{tabular}{|c|c|c|c|c|c|}
\hline \multirow[b]{2}{*}{ Variable } & \multirow[b]{2}{*}{$\begin{array}{l}\text { Control group (157) } \\
(\%)\end{array}$} & \multicolumn{2}{|c|}{ IVF } & \multirow[b]{2}{*}{$\begin{array}{l}\text { P-value } \\
\text { for all }\end{array}$} & \multirow{2}{*}{$\begin{array}{c}\text { P-value }^{\mathrm{a}} \\
\text { IVF Singleton/ } \\
\text { Multiple }\end{array}$} \\
\hline & & $\begin{array}{c}\text { Singleton }(77) \\
(\%)\end{array}$ & $\begin{array}{l}\text { Multiple (55) } \\
(\%)\end{array}$ & & \\
\hline Age $\leq 35$ years & $125(79.62)$ & $33(42.86)$ & $27(49.09)$ & $<0.0001$ & 0.4782 \\
\hline Age $\geq 35$ years & $32(20.38)$ & $44(57.14)$ & $28(50.91)$ & & \\
\hline Multiparous & $71(45.22)$ & $10(12.99)$ & $32(58.18)$ & $<0.0001$ & $<0.0001$ \\
\hline Nulliparous & $86(54.78)$ & $67(87.01)$ & $23(41.82)$ & & \\
\hline Embryos: not used (control group) & $157(100.00)$ & $0(0.00)$ & $0(0.00)$ & $<0.0001$ & 0.1336 \\
\hline Fresh embryos & $0(0.00)$ & $61(79.22)$ & $49(89.09)$ & & \\
\hline Cryopreserved embryos & $0(0.00)$ & $16(20.78)$ & $6(10.91)$ & & \\
\hline Embryo transfer, 0 & $157(100.00)$ & $0(0.00)$ & $0(0.00)$ & $<0.0001$ & $<0.0001$ \\
\hline Embryo transfer, 1 & $0(0.00)$ & $53(68.83)$ & $2(3.64)$ & & \\
\hline Embryo transfer, $\geq 2$ & $0(0.00)$ & $24(31.17)$ & $53(96.36)$ & & \\
\hline
\end{tabular}

All data are expressed as $\mathrm{n}(\%) . \mathrm{P}<0.0001$, highly significant. P-values according to ${ }^{\mathrm{a} C h i-s q u a r e}$ test. IVF, in vitro fertilization.

mean GSA and average birth weight were significantly lower in the IVF group (in singleton pregnancies -37.97 weeks and $\sim 3,002 \mathrm{~g}$ ), especially for multiple pregnancies (35.87 weeks and $\sim 2,289$ g respectively), compared to spontaneously obtained pregnancies (38.88 weeks and $\sim 3,260 \mathrm{~g}$ ) (Table I).

Table II shows that $\sim 50 \%$ of the pregnant women in the IVF group were $>35$ years of age $(57.14 \%$ in singleton pregnancies group and $50.91 \%$ in multiple pregnancies group) compared with the control group $(20.38 \%, \mathrm{P}<0.0001)$. Additionally, pregnant women who obtained a singleton pregnancy through IVF tended to be nulliparous $(87.01 \%)$, compared to those who obtained a multiple pregnancy $(41.82 \%, \mathrm{P}<0.0001)$.

The pregnancy distribution according to the type of embryo used, i.e., fresh vs. cryopreserved was analyzed (Table II). The results showed that IVF pregnancies were obtained predominantly by using fresh embryos, with a higher frequency in multiple pregnancies than singleton ones (89.09 vs. $79.22 \%$,
$\mathrm{P}=0.1336)$. The use of cryopreserved embryos was more frequently reported in singleton pregnancies than multiple pregnancies ( 20.78 vs. $10.91 \%, \mathrm{P}=0.1336$ ).

In $>30 \%$ of IVF singleton pregnancies, 2 or more embryos were initially transferred, resulting in a single pregnancy (Table II). For this reason, whether or not the loss or underdevelopment of an embryo was an independent risk factor for complications, was assessed. However, no significant difference for any complication analyzed was identified.

Furthermore, the possibility that the loss of an embryo was caused by risk factors associated with the IVF procedure, such as maternal age ( $\geq 35$ years), nulliparity, type of embryo used (fresh or cryopreserved) was assessed; however, no conclusive evidence for this hypothesis was obtained. The results were not statistically significant for maternal age ( $\geq 35$ years; $P=0.2558$ ), nulliparous women $(\mathrm{P}=0.9318)$, or type of embryo used, i.e., fresh/frozen $(\mathrm{P}=0.2222)$ (Table III). 
Table III. Analysis of the risk factors that can influence the loss of an embryo in IVF pregnancies.

\begin{tabular}{|c|c|c|c|}
\hline No. of embryo transfer & Age 35 years $(\%)$ & Age $\geq 35$ years $(\%)$ & P-value \\
\hline 1 & $25(47.17)$ & $28(52.83)$ & 0.2558 \\
\hline 2 & $8(33.33)$ & $16(66.67)$ & \\
\hline No. of embryo transfer & Multiparous (\%) & Nuliparous (\%) & P-value ${ }^{a}$ \\
\hline 1 & $7(13.21)$ & $46(86.79)$ & 0.9318 \\
\hline 2 & $3(12.50)$ & $21(87.50)$ & \\
\hline No. of embryo transfer & Fresh embryos (\%) & Cryopreserved embryos (\%) & P-value \\
\hline 1 & $44(83.02)$ & $9(16.98)$ & 0.2222 \\
\hline 2 & $17(70.83)$ & $7(29.17)$ & \\
\hline
\end{tabular}

All data are expressed as $\mathrm{n}(\%)$. $\mathrm{P}<0.05$, significant; $\mathrm{P}<0.001$, highly significant. $\mathrm{P}$-values according to ${ }^{\mathrm{a}}$ Chi-square test. IVF, in vitro fertilization.

Obstetric and neonatal complications. Obstetric and neonatal complications in the IVF group compared with the spontaneous conception group are described in Table IV.

Variables showing obstetric and neonatal complications among the three groups were analyzed to identify for which there was a possible influence determined either by the use of IVF or by the type of pregnancy, i.e., single and multiple, respectively.

Regarding obstetric complications, women who obtained a pregnancy through IVF, compared with spontaneous conception, had an increased rate of pregnancy-induced hypertension $(\mathrm{P}<0.0001)$. In the IVF group, this complication was more common in multiple pregnancies compared with singleton ones (18.18 vs. $6.49 \%$ ). No statistically significant difference was demonstrated between the groups for gestational diabetes $(\mathrm{P}=0.6139)$ and placental abnormalities $(\mathrm{P}=0.0695)$.

Regarding neonatal complications, the proportion of prematurity, LBW, SGA and LGA was significantly higher in the IVF group compared with the spontaneous conception group, except for LGA, which occurred only in singleton IVF pregnancies. No differences were demonstrated between the groups for IUGR $(\mathrm{P}=0.0507)$ and high birth weight $(\mathrm{P}=0.2559)$.

Although none of the pregnancies obtained by spontaneous conception had an Apgar score $<7$ each $1 \mathrm{~min}$, it cannot be said that pregnancies obtained by IVF have a risk of hypoxia at birth, as no statistically significant difference was demonstrated $(\mathrm{P}=0.0702)$.

Neonates obtained through IVF were more frequently admitted in NICU, compared with those obtained spontaneously $(\mathrm{P}<0.0001)$. Admission of the neonate in NICU was more common in the case of multiple pregnancies, compared with singleton pregnancies (67.27 vs. $33.77 \%$ ).

Given that the rate of prematurity was higher in the IVF group, this was closely correlated with the frequency of respiratory distress syndrome in these neonates $(\mathrm{P}<0.0001)$. As for the other complications analysed, the RDS rate was higher for multiple pregnancies compared with singleton ones (60.00 vs. $18.18 \%)$. For this reason, the need for oxygen therapy was higher in the IVF vs. control group $(\mathrm{P}<0.0001)$, but the need for exogenous surfactant administration was not statistically significant among the groups $(\mathrm{P}=0.3816)$.

Regarding the need of respiratory support, it was observed that neonates obtained by IVF required more frequently non-invasive ventilation with T-piece system compared to those obtained spontaneously $(\mathrm{P}<0.0001)$. Nasal CPAP and invasive ventilation were used with similar frequency within the two groups $(\mathrm{P}=0.0812$, respectively $\mathrm{P}=0.0695)$.

The incidence of apnea of prematurity did not show statistically significant differences between the groups $(\mathrm{P}=0.2079)$, but IVF neonates showed more frequent arterial hypotension compared to those obtained spontaneously $(\mathrm{P}<0.0001)$. This complication occurred more frequently in multiple pregnancies, compared with singletons in the IVF group (30.91 vs. 12.99\%).

Analysis of neonates with congenital anomalies, including congenital heart abnormalities did not show statistically significant differences between IVF pregnancies compared with the control group $(\mathrm{P}=0.1727$ and $\mathrm{P}=0.6608)$.

The results showed that neonates obtained through IVF, especially those from multiple pregnancies, required more frequently parenteral nutrition $(\mathrm{P}<0.0001)$, antibiotic therapy $(\mathrm{P}<0.0001)$ and vasoactive drugs $(\mathrm{P}<0.0001)$. The use of blood transfusions did not show significant differences between the study groups $(\mathrm{P}=0.0568)$.

Analysis of obstetric and neonatal complications in the IVF group vs. spontaneous conception stratified by the type of embryo used: fresh vs. cryopreserved. An analysis of whether the type of embryo used could have any influence on the occurrence of obstetric and neonatal complications was performed using the Fisher test. The results were not statistically significant for most of the complications studied (P>0.05) (Table V).

Analysis of obstetric and neonatal complications in the IVF group vs. spontaneous conception stratified by parity and 
Table IV. Analysis of obstetric and neonatal complications in the IVF group vs. spontaneous conception by birth plurality.

\begin{tabular}{|c|c|c|c|c|c|}
\hline \multirow[b]{2}{*}{ Variable } & \multirow[b]{2}{*}{$\begin{array}{c}\text { Control group (157) } \\
(\%)\end{array}$} & \multicolumn{2}{|c|}{ IVF } & \multirow[b]{2}{*}{$\begin{array}{l}\text { P-value } \\
\text { for all }\end{array}$} & \multirow{2}{*}{$\begin{array}{l}\text { P-value }^{\mathrm{a}} \\
\text { IVF Singleton/ } \\
\text { Multiple }\end{array}$} \\
\hline & & $\begin{array}{c}\text { Singleton }(77) \\
(\%)\end{array}$ & $\begin{array}{c}\text { Multiple (55) } \\
(\%)\end{array}$ & & \\
\hline \multicolumn{6}{|l|}{ Maternal complications } \\
\hline Pregnancy-induced hypertension & $3(1.91)$ & $5(6.49)$ & $10(18.18)$ & $<0.0001$ & 0.0370 \\
\hline Gestational diabetes & $6(3.82)$ & $5(6.49)$ & $2(3.64)$ & 0.6139 & 0.4702 \\
\hline Placental anomalies & $2(1.27)$ & $4(5.19)$ & $4(7.27)$ & 0.0695 & 0.6218 \\
\hline \multicolumn{6}{|l|}{ Neonatal complications } \\
\hline Prematurity & $10(6.37)$ & $13(16.88)$ & $29(52.73)$ & $<0.0001$ & $<0.0001$ \\
\hline LBW & $7(4.46)$ & $14(18.18)$ & $38(69.09)$ & $<0.0001$ & $<0.0001$ \\
\hline SGA & $13(8.28)$ & $11(14.29)$ & $21(38.18)$ & $<0.0001$ & 0.0016 \\
\hline IUGR & $3(1.91)$ & $5(6.49)$ & $0(0.00)$ & 0.0507 & 0.0540 \\
\hline LGA & $12(7.64)$ & $9(11.69)$ & $0(0.00)$ & 0.0373 & 0.0086 \\
\hline High birth weight & $6(3.82)$ & $4(5.19)$ & $0(0.00)$ & 0.2559 & 0.0861 \\
\hline Apgar score $<7$ per $1 \mathrm{~min}$ & $0(0.00)$ & $1(1.30)$ & $2(3.64)$ & 0.0702 & 0.374 \\
\hline NICU & $12(7.64)$ & $26(33.77)$ & $37(67.27)$ & $<0.0001$ & 0.00014 \\
\hline RDS & $10(6.37)$ & $14(18.18)$ & $33(60.00)$ & $<0.0001$ & $<0.0001$ \\
\hline Apnea of prematurity & $2(1.27)$ & $4(5.19)$ & $2(3.64)$ & 0.2079 & 0.6717 \\
\hline Arterial hypotension & $7(4.46)$ & $10(12.99)$ & $17(30.91)$ & $<0.0001$ & 0.0118 \\
\hline Cardiac anomalies & $14(8.92)$ & $9(11.69)$ & $7(12.73)$ & 0.6608 & 0.8569 \\
\hline Congenital anomalies & $1(0.64)$ & $3(3.90)$ & $2(3.64)$ & 0.1727 & 0.9386 \\
\hline Oxygen therapy & $10(6.37)$ & $20(25.97)$ & $35(63.64)$ & $<0.0001$ & $<0.0001$ \\
\hline Exogenous surfactant & $2(1.27)$ & $3(3.90)$ & $2(3.64)$ & 0.3816 & 0.9386 \\
\hline T-piece system & $3(1.91)$ & $8(10.39)$ & $12(21.82)$ & $<0.0001$ & 0.0710 \\
\hline Nasal CPAP & $2(1.27)$ & $5(6.49)$ & $3(5.45)$ & 0.0812 & 0.8052 \\
\hline Mechanical ventilation & $2(1.27)$ & $4(5.19)$ & $4(7.27)$ & 0.0695 & 0.6218 \\
\hline Parenteral nutrition & $12(7.64)$ & $17(22.08)$ & $27(49.09)$ & $<0.0001$ & 0.0012 \\
\hline Antibiotic therapy & $9(5.73)$ & $12(15.58)$ & $26(47.27)$ & $<0.0001$ & $<0.0001$ \\
\hline Vasoactive drugs & $6(3.82)$ & $7(9.09)$ & $14(25.45)$ & $<0.0001$ & $<0.0001$ \\
\hline Blood transfusions & $3(1.91)$ & $3(3.90)$ & $5(9.09)$ & 0.0568 & 0.2175 \\
\hline
\end{tabular}

All data are expressed as $\mathrm{n}(\%) . \mathrm{P}<0.05$, significant; $\mathrm{P}<0.001$, highly significant. $\mathrm{P}$-values according to a Chi-square test. IVF, in vitro fertilization; LBW, low birth weight; SGA, small for gestational age; IUGR, intrauterine growth restriction; LGA, large for gestational age; NICU, Neonatal Intensive Care Unit; RDS, respiratory distress syndrome; Nasal CPAP, nasal continuous positive airway pressure.

maternal age. The impact of IVF, nulliparity and advanced maternal age on the occurrence of obstetric and neonatal complications was assessed by performing multivariable logistical analysis. The result of the analysis was expressed, for each variable, as the odds Ratio $(\mathrm{OR})+95 \%$ confidence interval of OR and P-value (Table VI).

Nulliparity, as a confounding variable, influenced NICU admission $(\mathrm{OR}=0.46,95 \% \mathrm{CI}=0.25-0.88), \mathrm{LBW}(\mathrm{OR}=0.37$, $95 \% \mathrm{CI}=0.19-0.75)$, the need of parenteral nutrition $(\mathrm{OR}=0.49$, 95\% CI $=0.26-0.94)$ and antibiotic therapy $(\mathrm{OR}=0.48$, $95 \% \mathrm{CI}=0.25-0.97$ ), but age $\geq 35$ years had no influence as a confounding variable.

During our analysis, it was found that women who underwent IVF treatment were 6.42-fold more likely to develop pregnancy-induced hypertension $(\mathrm{OR}=6.42,95 \% \mathrm{CI}=1.72-23.92)$ and 5.49-fold more likely to develop placental abnormalities $(\mathrm{OR}=5.49,95 \% \mathrm{CI}=1.07-28.17)$.
Neonates obtained by IVF procedures were 6.52-fold more predisposed to prematurity $(\mathrm{OR}=6.52$, 95\% CI=2.99-14.20), 17.18-fold more likely to have LBW $(\mathrm{OR}=17.18,95 \% \mathrm{CI}=7.06-41.87)$ and 4.10 -fold more likely to be $\mathrm{SGA}(\mathrm{OR}=4.10,95 \% \mathrm{CI}=1.95-8.59)$. In addition, these neonates were 11.91-fold more likely to be admitted in NICU $(\mathrm{OR}=11.91$, 95\% $\mathrm{CI}=5.72-24.81)$. RDS was 8.71 -fold more likely to occur in the IVF group ( $\mathrm{OR}=8.71$, $95 \% \mathrm{CI}=4.00-18.95)$, that required oxygen therapy $(\mathrm{OR}=11.19$, 95\% CI=5.16-24.26), non-invasive with a T-piece system $(\mathrm{OR}=7.88,95 \% \mathrm{CI}=2.17-28.57)$ and nasal CPAP $(\mathrm{OR}=5.79$, 95\% CI=1.14-29.43).

IVF neonates were 6.57 time more likely to require parenteral nutrition $(\mathrm{OR}=6.57,95 \% \mathrm{CI}=3.14-13.76)$ and antibiotic therapy $(\mathrm{OR}=6.94,95 \% \mathrm{CI}=3.06-15.72)$. As IVF patients are 4.16-fold more likely to develop neonatal arterial hypotension $(\mathrm{OR}=4.16,95 \% \mathrm{CI}=1.66-10.46)$, they are 3.44-fold more 
Table V. Analysis of obstetric and neonatal complications in the IVF group vs. spontaneous conception stratified by the type of embryo used: fresh vs. cryopreserved.

\begin{tabular}{lccc}
\hline Variable & Fresh embryos (110) $(\%)$ & Cryopreserved embryos (22) (\%) & P-value $^{\mathrm{a}}$ \\
\hline Maternal complications & & & \\
Pregnancy-induced hypertension & $12(10.91)$ & $3(13.64)$ & 0.4937 \\
Gestational diabetes & $4(3.64)$ & $3(13.64)$ & 0.0754 \\
Placental anomalies & $6(5.45)$ & $2(9.09)$ & 0.4912 \\
Neonatal complications & & & \\
Prematurity & $35(31.82)$ & $7(31.82)$ & 0.6062 \\
LBW & $45(40.91)$ & $7(31.82)$ & 0.2917 \\
SGA & $28(25.45)$ & $4(18.18)$ & 0.3349 \\
IUGR & $3(2.73)$ & $2(9.09)$ & 0.1612 \\
LGA & $8(7.27)$ & $1(4.55)$ & 0.5383 \\
High birth weight & $4(3.64)$ & $0(0.00)$ & 0.4778 \\
Apgar score $<7$ per 1 min & $1(0.91)$ & $2(9.09)$ & 0.0719 \\
NICU & $53(48.18)$ & $10(45.45)$ & 0.5010 \\
RDS & $39(35.45)$ & $8(36.36)$ & 0.6325 \\
Apnea of prematurity & $3(2.73)$ & $3(13.64)$ & 0.0579 \\
Arterial hypotension & $22(20.00)$ & $5(22.73)$ & 0.524 \\
Cardiac anomalies & $12(10.91)$ & $4(18.18)$ & 0.2077 \\
Congenital anomalies & $4(3.64)$ & $1(4.55)$ & 0.8063 \\
Oxygen therapy & $47(42.73)$ & $8(36.36)$ & 0.3795 \\
Exogenous surfactant & $2(1.82)$ & $3(13.64)$ & 0.0325 \\
T-piece system & $15(13.64)$ & $5(22.73)$ & 0.2432 \\
Nasal CPAP & $4(3.64)$ & $4(18.18)$ & 0.0263 \\
Mechanical ventilation & $5(4.55)$ & $3(13.64)$ & 0.1024 \\
Parenteral nutrition & $36(32.73)$ & $8(36.36)$ & 0.5281 \\
Antibiotic therapy & $31(28.18)$ & $7(31.82)$ & 0.5290 \\
Vasoactive drugs & $17(15.45)$ & $4(18.18)$ & 0.5001 \\
Blood transfusions & $6(5.45)$ & $2(9.09)$ & 0.4912 \\
\hline Al & & & \\
\hline
\end{tabular}

All data are expressed as $\mathrm{n}(\%)$. $\mathrm{P}<0.05$, significant; $\mathrm{P}<0.001$, highly significant. $\mathrm{P}$-values according to ${ }^{\mathrm{a}}$ Fisher test. IVF, in vitro fertilization; LBW, low birth weight; SGA, small for gestational age; IUGR, intrauterine growth restriction; LGA, large for gestational age; NICU, Neonatal Intensive Care Unit; RDS, respiratory distress syndrome; Nasal CPAP, nasal continuous positive airway pressure.

likely to require vasoactive medication $(\mathrm{OR}=3.44,95 \%)$. $\mathrm{CI}=1.26-9.41)$.

The results also showed that, although a simple analysis between groups did not reach the $95 \%$ significance threshold $(\mathrm{P}<0.05)$ for placental anomalies and the need of nasal CPAP (in which case $\mathrm{P}>0.05$, but $\mathrm{P}<0.1-90 \%$ significance threshold), ART significantly influenced the risk of these complications when other factors were taken into account in a multivariable analysis.

\section{Discussion}

In the present study, we demonstrated that pregnancies obtained through in vitro fertilization (IVF) have a higher risk of complications compared with pregnancies obtained spontaneously, which correlates with data from the literature.

Over time, research has shown that some pregnancies obtained through assisted reproduction techniques (ARTs) have a higher risk of pregnancy-induced hypertension (9-11), gestational diabetes $(8,12,13)$ and placental anomalies $(14,15)$. Complications can also affect the neonates, who are at risk of prematurity $(2,3)$, low birth weight $(\mathrm{LBW})(2,4)$, small for gestational age (SGA) (2), large for gestational age (LGA) (16-18) and congenital anomalies (2-4).

The results of the present study showed that women who become pregnant by IVF have a higher risk of developing pregnancy-induced hypertension and placental abnormalities. In a population-based cohort study, Opdahl et al compared pregnancies obtained by ART with pregnancies obtained spontaneously and showed an increased risk for hypertensive disorders in pregnancy in the ART group (9). In the present study, this effect could have been explained by the increased proportion of women over the age of 35 years, but the multivariable analysis revealed that advanced maternal age had no influence on the risk of pregnancy complications. In addition, given that an increased proportion of women with multiple IVF pregnancies developed pregnancy-induced hypertension, we consider that multiple pregnancies are an 
Table VI. Multivariable analysis of obstetric and neonatal complications in the nulliparous group.

\begin{tabular}{|c|c|c|c|c|c|c|}
\hline \multirow[b]{2}{*}{ Variable } & \multicolumn{3}{|c|}{ Main variable: control vs. IVF group } & \multicolumn{3}{|c|}{ Confounding factor: Nulliparous } \\
\hline & $\begin{array}{l}\text { Odds } \\
\text { ratio }\end{array}$ & $95 \% \mathrm{CI}$ & $\begin{array}{c}\text { P-value } \\
\text { Chi }^{2} \text { Wald }\end{array}$ & $\begin{array}{c}\text { Odds } \\
\text { ratio }\end{array}$ & $95 \% \mathrm{CI}$ & $\begin{array}{c}\text { P-value } \\
\text { Chi }^{2} \text { Wald }\end{array}$ \\
\hline \multicolumn{7}{|l|}{ Maternal complications } \\
\hline Pregnancy-induced hypertension & 6.42 & $1.72-23.92$ & 0.0056 & 0.60 & $0.22-1.64$ & 0.3261 \\
\hline Gestational diabetes & 1.35 & $0.41-4.50$ & 0.6223 & 0.37 & $0.12-1.20$ & 0.0986 \\
\hline Placental anomalies & 5.49 & $1.07-28.17$ & 0.0410 & 1.25 & $0.31-5.01$ & 0.7556 \\
\hline \multicolumn{7}{|l|}{ Neonatal complications } \\
\hline Prematurity & 6.52 & $2.99-14.20$ & $<0.0001$ & 0.70 & $0.36-1.36$ & 0.2940 \\
\hline LBW & 17.18 & $7.06-41.87$ & $<0.0001$ & 0.37 & $0.19-0.75$ & 0.0052 \\
\hline SGA & 4.10 & $1.95-8.59$ & 0.0002 & 0.69 & $0.36-1.37$ & 0.2949 \\
\hline IUGR & 2.39 & $0.51-11.26$ & 0.2712 & 4.16 & $0.50-34.53$ & 0.1865 \\
\hline LGA & 1.18 & $0.46-3.04$ & 0.7362 & 0.53 & $0.22-1.31$ & 0.1683 \\
\hline High birth weight & 1.17 & $0.31-4.48$ & 0.8147 & 0.24 & $0.06-0.99$ & 0.0480 \\
\hline Apgar score $<7$ per $1 \mathrm{~min}$ & - & - & 0.9954 & 0.81 & $0.07-9.52$ & 0.8714 \\
\hline NICU & 11.91 & $5.72-24.81$ & $<0.0001$ & 0.46 & $0.25-0.88$ & 0.0186 \\
\hline RDS & 8.71 & $4.00-18.95$ & $<0.0001$ & 0.54 & $0.28-1.04$ & 0.0664 \\
\hline Apnea of prematurity & 4.00 & $0.73-21.95$ & 0.1111 & 1.65 & $0.32-8.48$ & 0.5432 \\
\hline Arterial hypotension & 4.16 & $1.66-10.46$ & 0.0024 & 1.15 & $0.52-2.56$ & 0.7292 \\
\hline Cardiac anomalies & 1.47 & $0.65-3.33$ & 0.3524 & 0.49 & $0.23-1.07$ & 0.0769 \\
\hline Congenital anomalies & 4.55 & $0.46-44.61$ & 0.1936 & 1.03 & $0.18-5.96$ & 0.9682 \\
\hline Oxygen therapy & 11.19 & $5.16-24.26$ & $<0.0001$ & 0.52 & $0.28-1.01$ & 0.0519 \\
\hline Exogenous surfactant & 3.93 & $0.70-21.99$ & 0.1189 & 0.20 & $0.04-1.10$ & 0.0639 \\
\hline T-piece system & 7.88 & $2.17-28.57$ & 0.0017 & 0.59 & $0.24-1.49$ & 0.2691 \\
\hline Nasal CPAP & 5.79 & $1.14-29.43$ & 0.0344 & 0.77 & $0.21-2.88$ & 0.7088 \\
\hline Mechanical ventilation & 4.29 & $0.82-22.39$ & 0.0845 & 0.77 & $0.21-2.91$ & 0.7106 \\
\hline Parenteral nutrition & 6.57 & 3.14-13.76 & $<0.0001$ & 0.49 & $0.26-0.94$ & 0.0318 \\
\hline Antibiotic therapy & 6.94 & $3.06-15.72$ & $<0.0001$ & 0.48 & $0.25-0.97$ & 0.0414 \\
\hline Vasoactive drugs & 3.44 & $1.26-9.41$ & 0.0162 & 0.91 & $0.39-2.18$ & 0.8438 \\
\hline Blood transfusions & 3.08 & 0.74-12.89 & 0.1234 & 0.64 & $0.19-2.23$ & 0.4890 \\
\hline
\end{tabular}

IVF, in vitro fertilization; LBW, low birth weight; SGA, small for gestational age; IUGR, intrauterine growth restriction; LGA, large for gestational age; NICU, Neonatal Intensive Care Unit; RDS, respiratory distress syndrome; Nasal CPAP, nasal continuous positive airway pressure; CI, confidence interval. $\mathrm{P}<0.05$, significant; $\mathrm{P}<0.001$, highly significant.

independent risk factor, especially if the mother is $>35$ years of age (19).

Two recent meta-analyses compared singleton IVF pregnancies with spontaneous conception and demonstrated an increased risk of placental anomalies associated with IVF $(14,20)$. Karami et al demonstrated that the risk of placental abnormalities was identified for multiple pregnancies obtained by IVF (15), but this aspect was not demonstrated in the present study.

Bosdou et al demonstrated in a recent meta-analysis that using IVF compared with intracytoplasmic sperm insemination (ICSI) increases the risk of developing gestational diabetes (GD) (21). In this study, the results were inconsistent with the data reported in the literature $(8,12,13)$. Even after adjustment for confounding factors (advanced maternal age, nulliparity), having an IVF pregnancy did not increase the risk of GD.
Consistent with the results of previous studies $(2,3)$, the prematurity rate was higher in the IVF group, compared with the control group. A recent meta-analysis, which included 52 cohort studies, with a total of 181,741 single pregnancies obtained by IVF/ICSI and approximately 4.6 million spontaneous neonates worldwide showed that pregnancies obtained by ART had increased risk of prematurity, LBW, congenital anomalies and perinatal mortality (2). Given that $41.6 \%$ of all IVF pregnancies were multiple pregnancies, we consider that the increased incidence of prematurity can be a side effect of these high-risk pregnancies. To verify this hypothesis, we performed a stratified analysis of data according to the type of pregnancy (singleton/multiple). Thus, after the analysis was restricted to singleton pregnancies, we observed a reduction in the incidence of complications for most of the variables studied. This conclusion has been demonstrated in previous studies (22-25). 
The increased incidence of prematurity in the present study was also correlated with changes in birth weight. Thus, we observed that neonates obtained by IVF have a higher risk of LBW and SGA, compared to those obtained spontaneously. This may be associated with the increased rate of multiple pregnancies in the IVF population, but Schieve et al demonstrated that the risk was also observed for singleton ART pregnancies (4). However, the associated maternal complications such as pregnancy-induced hypertension and placental abnormalities, as well as the culture medium used for embryo development, could have influenced birth weight.

For LGA, and high birth weight, there were no differences observed between the groups. Maheshwari et al demonstrated that the transfer of cryopreserved embryos reduced the risk of prematurity, LBW and SGA, but increased the incidence of LGA and high birth weight (16). A retrospective study showed that transferring cryopreserved embryos increased the birth weight with an average of $142.3 \mathrm{~g}$. The authors of that study concluded that the cryopreservation group had a 1.7-fold higher risk of high birth weight (17). The analysis of our data did not show an increased risk of LGA $(\mathrm{P}=0.5383)$, or high birth weight $(\mathrm{P}=0.4778)$, when using cryopreservation techniques (Table $\mathrm{V})$.

Results of the present study did not demonstrate an increased risk for congenital anomalies associated with IVF. This is consistent with literature data, as previous findings have shown that the use of ICSI increases the risk of congenital anomalies compared to IVF (26).

Given the increased incidence of prematurity in the IVF group, the neonates included in our study had general prematurity complications. Thus, they were more likely to be hospitalized in NICU for respiratory complications such as respiratory distress syndrome (RDS), for which they needed oxygen therapy and non-invasive respiratory support. The risk of arterial hypotension may be secondary to both prematurity and placental abnormalities that may be associated with maternal bleeding and the risk of neonatal hypovolemia.

The risk of pregnancy complications is directly proportional to maternal age (27). It is considered that women $>35$ years of age may associate other comorbidities in addition to the etiology of infertility. This can influence the evolution of IVF pregnancies, including placing the pregnant woman in a risk category (28). For this reason, a data analysis was performed according to the presence of nulliparity and advanced maternal age, but the results were not significant for most of the complications analysed. However, advanced maternal age and nulliparity are risk factors that predispose to the use of IVF anyway and this may be the reason that the complications analyzed were not influenced. Wennberg et al demonstrated in a retrospective study that advanced maternal age increased the risk of complications in pregnancy, regardless of the type of conception, i.e., spontaneous vs. assisted reproduction (29).

Given the increased rate of multiple pregnancies in the IVF group, it is necessary to implement single embryo transfer protocols. It is currently considered the most effective method to avoid multiple pregnancies and their associated complications (30-33). According to ESHRE records, the transfer of a single embryo in Romania increased from $16.9 \%$ in 2015 to $22.5 \%$ in $2016(1,34)$.

The use of cryopreservation allows the selection of high-quality embryos and the transfer of a single embryo, which helps reduce the incidence of multiple pregnancies and thus the associated complications. Given the benefits reported after the transfer of cryopreserved embryos, the implementation of the freeze-all strategy is being discussed (35-37). The reported benefits include a reduction in the overall incidence of ovarian hyperstimulation syndrome and the incidence of prematurity, LBW and VLBW. Simultaneously, recent findings have shown an increased risk of pregnancy-induced hypertensive conditions, including pre-eclampsia (38). The risk of complications calls into question the widespread use of the freeze-all transfer policy in the pregnancies obtained by ART $(16,39)$.

The present study has some limitations. First, its partial retrospective design, a single-center study, and the small cohort of patients are factors that may limit the generalization of our findings. Additionally, the lack of multiple pregnancies in the control group reduces the possibility to determine exactly whether they influence the prognosis of pregnancies obtained through IVF. Multicenter, prospective studies on large population groups from Romania are needed to demonstrate that IVF use increases the risk of pregnancy complications.

Second, data on the etiology of infertility were limited and could not be included. Further studies are needed to include information on the cause of infertility, on the ovarian stimulation protocol used, serum hormone levels during ovarian stimulation, as well as the embryonic stage at the time of transfer (cleavage or blastocyst stage).

Third, no information was obtained on the diagnosis of subfertility in the control population, which, according to the above-mentioned studies (6,7), can influence the results.

In conclusion, women who become pregnant through in vitro fertilization have a higher risk of complications in pregnancy. Multiple pregnancies can be considered a risk factor, but literature data show that singleton pregnancies obtained through IVF also present complications. Additional factors that could not be controlled in the present study (ovarian stimulation protocol, culture medium used, type of embryo transferred, population of subfertile women) may influence this risk.

Given the results of the present study, we recommend the implementation of single embryo transfer protocols to reduce the complications associated with multiple pregnancies.

\section{Acknowledgements}

Not applicable.

\section{Funding}

No funding was received.

\section{Availability of data and materials}

All data generated or analyzed during this study are included in this published article.

\section{Authors' contributions}

AMB, SDP and SV designed the study. AMB and SDP consulted the literature and collected bibliographical data. 
AMB and SDP wrote the paper. SV reviewed and edited the manuscript. AMB and SV confirm the authenticity of all the raw data. All authors have read and approved the final manuscript for publication.

\section{Ethics approval and consent to participate}

The present study was approved by the Ethics Committee of Elias University Emergency Hospital (approval no. 7017). Written informed consent was obtained from each mother.

\section{Patient consent for publication}

Not applicable.

\section{Competing interests}

The authors declare that they have no competing interests.

\section{References}

1. European IVF-Monitoring Consortium (EIM) $\$$ for the European Society of Human Reproduction and Embryology (ESHRE); Wyns C, Bergh C, Calhaz-Jorge C, De Geyter C, Kupka MS, Motrenko T, Rugescu I, Smeenk J, Tandler-Schneider A, et al: ART in Europe, 2016: Results generated from European registries by ESHRE. Hum Reprod Open 2020: hoaa032, 2020.

2. Qin JB, Sheng XQ, Wu D, Gao SY, You YP, Yang TB and Wang H: Worldwide prevalence of adverse pregnancy outcomes among singleton pregnancies after in vitro fertilization/intracytoplasmic sperm injection: A systematic review and meta-analysis. Arch Gynecol Obstet 295: 285-301, 2017.

3. Helmerhorst FM, Perquin DA, Donker D and Keirse MJ: Perinatal outcome of singletons and twins after assisted conception: A systematic review of controlled studies. BMJ 328: 261, 2004.

4. Schieve LA, Meikle SF, Ferre C, Peterson HB, Jeng G and Wilcox LS: Low and very low birth weight in infants conceived with use of assisted reproductive technology. N Engl J Med 346 731-737, 2002.

5. Hayashi M, Nakai A, Satoh S and Matsuda Y: Adverse obstetric and perinatal outcomes of singleton pregnancies may be related to maternal factors associated with infertility rather than the type of assisted reproductive technology procedure used. Fertil Steril 98: 922-928, 2012.

6. Marino JL, Moore VM, Willson KJ, Rumbold A, Whitrow MJ, Giles LC and Davies MJ: Perinatal outcomes by mode of assisted conception and sub-fertility in an Australian data linkage cohort. PLoS One 9: e80398, 2014

7. Jaques AM, Amor DJ, Baker HW, Healy DL, Ukoumunne OC, Breheny S, Garrett C and Halliday JL: Adverse obstetric and perinatal outcomes in subfertile women conceiving without assisted reproductive technologies. Fertil Steril 94: 2674-2679, 2010.

8. Lei LL, Lan YL, Wang SY, Feng W and Zhai ZJ: Perinatal complications and live-birth outcomes following assisted reproductive technology: A retrospective cohort study. Chin Med J (Engl) 132: 2408-2416, 2019.

9. Opdahl S, Henningsen AA, Tiitinen A, Bergh C, Pinborg A, Romundstad PR, Wennerholm UB, Gissler M, Skjærven R and Romundstad LB: Risk of hypertensive disorders in pregnancies following assisted reproductive technology: A cohort study from the CoNARTaS group. Hum Reprod 30: 1724-1731, 2015.

10. Thomopoulos C, Tsioufis C, Michalopoulou H, Makris T, Papademetriou V and Stefanadis C: Assisted reproductive technology and pregnancy-related hypertensive complications: A systematic review. J Hum Hypertens 27: 148-157, 2013.

11. Luke B, Brown MB, Eisenberg ML, Callan C, Botting BJ, Pacey A, Sutcliffe AG and Baker VL: In vitro fertilization and risk for hypertensive disorders of pregnancy: Associations with treatment parameters. Am J Obstet Gynecol 222: 350.e1-350.e13, 2020 .
12. Qin J, Liu X, Sheng X, Wang H and Gao S: Assisted reproductive technology and the risk of pregnancy-related complications and adverse pregnancy outcomes in singleton pregnancies: A meta-analysis of cohort studies. Fertil Steril 105: 73-85.e1, 6, 2016.

13. Zhu L, Zhang Y, Liu Y, Zhang R, Wu Y, Huang Y, Liu F, Li M, Sun S, Xing L, et al: Maternal and live-birth outcomes of pregnancies following assisted reproductive technology: A retrospective cohort study. Sci Rep 6: 35141, 2016.

14. Jackson RA, Gibson KA, Wu YW and Croughan MS: Perinatal outcomes in singletons following in vitro fertilization: A meta-analysis. Obstet Gynecol 103: 551-563, 2004.

15. Karami M, Jenabi E and Fereidooni B: The association of placenta previa and assisted reproductive techniques: A meta-analysis. J Matern Fetal Neonatal Med 31: 1940-1947, 2018.

16. Maheshwari A, Pandey S, Raja EA, Shetty A, Hamilton M and Bhattacharya S: Is frozen embryo transfer better for mothers and babies? Can cumulative meta-analysis provide a definitive answer? Hum Reprod Update 24: 35-58, 2018.

17. Litzky JF, Boulet SL, Esfandiari N, Zhang Y, Kissin DM, Theiler RN and Marsit CJ: Effect of frozen/thawed embryo transfer on birthweight, macrosomia, and low birthweight rates in US singleton infants. Am J Obstet Gynecol 218: 433.e1-433. e10, 2018.

18. Orvieto R, Kirshenbaum $\mathrm{M}$ and Gleicher $\mathrm{N}$ : Is embryo cryopreservation causing macrosomia-and what else? Front Endocrinol (Lausanne) 11: 19, 2020.

19. Avnon T, Ovental A and Many A: Twin versus singleton pregnancy in women $\geq 45$ years of age: Comparison of maternal and neonatal outcomes. J Matern Fetal Neonatal Med 34: 201-206, 2021.

20. Vermey BG, Buchanan A, Chambers GM, Kolibianakis EM, Bosdou J, Chapman MG and Venetis CA: Are singleton pregnancies after assisted reproduction technology (ART) associated with a higher risk of placental anomalies compared with non-ART singleton pregnancies? A systematic review and meta-analysis. BJOG 126: 209-218, 2019.

21. Bosdou JK, Anagnostis P, Goulis DG, Lainas GT, Tarlatzis BC, Grimbizis GF and Kolibianakis EM: Risk of gestational diabetes mellitus in women achieving singleton pregnancy spontaneously or after ART: A systematic review and meta-analysis. Hum Reprod Update 26: 514-544, 2020.

22. Bhattacharya $S$ and Kamath MS: Reducing multiple births in assisted reproduction technology. Best Pract Res Clin Obstet Gynaecol 28: 191-199, 2014.

23. Sazonova A, Källen K, Thurin-Kjellberg A, Wennerholm UB and Bergh C: Neonatal and maternal outcomes comparing women undergoing two in vitro fertilization (IVF) singleton pregnancies and women undergoing one IVF twin pregnancy. Fertil Steril 99: 731-737, 2013.

24. Gupta R, Sardana P, Arora P, Banker J, Shah S and Banker M: Maternal and neonatal complications in twin deliveries as compared to singleton deliveries following in vitro fertilization. J Hum Reprod Sci 13: 56-64, 2020.

25. Van Heesch MM, Evers JL, Dumoulin JC, van der Hoeven MA, van Beijsterveldt CE, Bonsel GJ, Dykgraaf RH, van Goudoever JB, Koopman-Esseboom C, Nelen WL, et al: A comparison of perinatal outcomes in singletons and multiples born after in vitro fertilization or intracytoplasmic sperm injection stratified for neonatal risk criteria. Acta Obstet Gynecol Scand 93: 277-286, 2014.

26. Davies MJ, Moore VM, Willson KJ, Van Essen P, Priest K, Scott H, Haan EA and Chan A: Reproductive technologies and the risk of birth defects. N Engl J Med 366: 1803-1813, 2012.

27. Kahveci B, Melekoglu R, Evruke IC and Cetin C: The effect of advanced maternal age on perinatal outcomes in nulliparous singleton pregnancies. BMC Pregnancy Childbirth 18: 343, 2018.

28. Zollner U and Dietl J: Perinatal risks after IVF and ICSI. J Perinat Med 41: 17-22, 2013.

29. Wennberg AL, Opdahl S, Bergh C, Henningsen AK, Gissler M, Romundstad LB, Pinborg A, Tiitinen A, Skjærven R and Wennerholm UB: Effect of maternal age on maternal and neonatal outcomes after assisted reproductive technology. Fertil Steril 106: 1142-1149.e14, 2016.

30. De Geyter C: Single embryo transfer in all infertile couples treated with assisted reproduction produces excellent results and avoids multiple births. Swiss Med Wkly 151: w20499, 2021. 
31. Klenov VE, Boulet SL, Mejia RB, Kissin DM, Munch E, Mancuso A and Van Voorhis BJ: Live birth and multiple birth rates in US in vitro fertilization treatment using donor oocytes: A comparison of single-embryo transfer and double-embryo transfer. J Assist Reprod Genet 35: 1657-1664, 2018.

32. Bergh C, Kamath MS, Wang R and Lensen S: Strategies to reduce multiple pregnancies during medically assisted reproduction. Fertil Steril 114: 673-679, 2020.

33. Kamath MS, Mascarenhas M, Kirubakaran R and Bhattacharya S: Number of embryos for transfer following in vitro fertilisation or intra-cytoplasmic sperm injection. Cochrane Database Syst Rev 8: CD003416, 2020.

34. De Geyter C, Calhaz-Jorge C, Kupka MS, Wyns C, Mocanu E, Motrenko T, Scaravelli G, Smeenk J, Vidakovic S, Goossens V; European IVF-monitoring Consortium (EIM) for the European society of human reproduction and embryology (ESHRE): ART in Europe, 2015: Results generated from European registries by ESHRE. Hum Reprod Open 2020: hoz038, 2020.

35. Kalinderis M, Kalinderi K, Srivastava G and Homburg R: When should we freeze embryos? current data for fresh and frozen embryo replacement IVF cycles. Reprod Sci 28: 3061-3072, 2021.
36. Maheshwari A, Pandey S, Shetty A, Hamilton $M$ and Bhattacharya S: Obstetric and perinatal outcomes in singleton pregnancies resulting from the transfer of frozen thawed versus fresh embryos generated through in vitro fertilization treatment: A systematic review and meta-analysis. Fertil Steril 98: 368-377. e1-e9, 2012.

37. Maheshwari A, Raja EA and Bhattacharya S: Obstetric and perinatal outcomes after either fresh or thawed frozen embryo transfer: An analysis of 112,432 singleton pregnancies recorded in the human fertilisation and embryology authority anonymized dataset. Fertil Steril 106: 1703-1708, 2016.

38. Roque M, Haahr T, Geber S, Esteves SC and Humaidan P: Fresh versus elective frozen embryo transfer in IVF/ICSI cycles: A systematic review and meta-analysis of reproductive outcomes. Hum Reprod Update 25: 2-14, 2019.

39. Roque M, Valle M, Sampaio M and Geber S: Obstetric outcomes after fresh versus frozen-thawed embryo transfers: A systematic review and meta-analysis. JBRA Assist Reprod 22: 253-260, 2018. 